\title{
Análisis de los "cinco grandes" factores de la personalidad en alumnos de la Universidad de mayores de Almería
}

\author{
Ma del Carmen Pérez-Fuentes, José J. Gázquez y Ma del Mar Molero \\ Universidad de Almería (España)
}

\begin{abstract}
El objetivo del presente estudio es conocer a través de la aplicación del NEO-FFI las características de las personalidad (en función de variables como la edad, el género y el estado civil) de un grupo específico de mayores de 50 años, 263 personas, que forman parte de un programa de envejecimiento activo, como es, el Programa Universitario para Personas Mayores "Ciencia y Experiencia" de la Universidad de Almería. Así, en función del género vemos que sólo en el factor Neuroticismo se observan diferencias significativas, las mujeres presentan mayores niveles que los hombres, siendo considerable el tamaño del efecto $(d=0,61)$. Respecto al estado civil, el grupo de viudos, en el factor A (Amabilidad), son los que obtienen las puntuaciones más bajas, con diferencias significativas con respecto al grupo de los casados y los divorciados/separados. Finalmente, el análisis en función de la edad, encontramos diferencias significativas en los factores: Extraversión, Responsabilidad, Neuroticismo y Apertura; siendo baja la proporción de variabilidad total de la puntuación obtenida en cada uno de ellos atribuible a la variable edad.
\end{abstract}

Palabras clave: Factores, personalidad, universidad de mayores, variables sociodemográficas.

Analysis of the"Big Five" personality factors in students of the Older People's University of Almería. The goal of the present study is to determine, through the administration of the NEO-FFI, the personality characteristics (as a function of variables such as age, gender, and civil status) of a specific group of 263 people over 50 years of age, who are part of an active ageing program such as the University Program for Older People "Science and Experience" of the University of Almería. Thus, as a function of gender, we only observed significant differences in the factor Neuroticism, with the women presenting higher levels than the men, with a considerable effect size $(d=0.61)$. With regard to civil status, the group of widowed people obtained the lowest scores in Factor A (Agreeableness), with significant differences with regard to the groups of married people and of divorced/separated people. Lastly, in the analysis as a function of age, we found significant differences in the following factors: Extraversion, Responsibility, Neuroticism, and Openness; the proportion of total variability attributable to age in the score of each variable was low.

Key words: Factors, personality, older people's university, sociodemographic variables.

Correspondencia: $\mathrm{M}^{\mathrm{a}}$ del Carmen Pérez Fuentes. Facultad de Ciencias de la Educación, Enfermería y Fisioterapia. Universidad de Almería. Ctra. de Sacramento, s/n. C.P. 04120. Almería (España). E-mail: $\underline{\text { mpf412@ual.es }}$ 
Son diversas las perspectivas desde las que la psicología se acerca al estudio y la evaluación de la personalidad, tomando cada vez más auge el Modelo de los Cinco Factores. Este paradigma responde al intento de reducir los numerosos factores que componen el constructo personalidad (Catell, 1949, 1993) que dificultan la aplicación de cuestionarios para su evaluación. El fundador de esta corriente lo encontramos en McDougall (1932), aunque fue Norman (1963) quién proporcionó la primera denominación de los cinco grandes factores. Actualmente, existe consenso en afirmar que este modelo ofrece una taxonomía válida para congregar los diversos rasgos de personalidad (McCrae y Costa, 2008).

Entre los instrumentos más completos y empleados de esta corriente encontramos el NEO-PI (Costa y McCrae, 1985). Los factores que mide este cuestionario coinciden con los "Cinco Grandes", y son: Neuroticismo (N), Extraversión (E), Apertura a la experiencia (O), Amabilidad (A) y Responsabilidad (C). Este primer instrumento fue revisado dando lugar al NEO-PI-R (Costa y McCrae, 1992), y además, con el objetivo de ofrecer un instrumento de aplicación rápida Costa y McGrae (1989) diseñaron una forma abreviada: el NEO-FFI.

Desde entonces, son múltiples los estudios que han utilizado este instrumento en el análisis de la personalidad. Así, ha sido aplicado en diferentes culturas apoyando la hipótesis de la universalidad de los rasgos de personalidad, que a través del instrumento se analizan (McCrae y Terracciano, 2005), aunque en otros casos, se destaca la necesidad de elaborar baremos diferentes en función de la edad, el género y el nivel académico (de Miguel, 2005). Algunos, sólo consideran necesario la adaptación en relación con la edad (McCrae, Costa y Martin, 2005), o se matiza, poniendo de relieve el objetivo o finalidad con que se implementa la prueba, ya que si el propósito es conocer las diferencias de personalidad en función del género, la utilización de baremos diferentes suprimiría estas (McCrae, Martin y Costa, 2005).

Así, destinado fundamentalmente a la población adulta ha sido aplicado a diferentes grupos de edad, desde la adolescencia a la vejez. Aunque los resultados en el análisis estructural del cuestionario son iguales para todos los grupos de edad, hay investigadores que consideran algunos ítems de difícil comprensión para los adolescentes o no pertinentes, para esta etapa de la vida (McCrae, Costa y Martin, 2005) apareciendo versiones del mismo para adolescentes jóvenes como el NEO-PI-3 (Costa, McCrae y Martin, 2008) y el NEO-FFI-3 (McCrae y Costa, 2007), realizándose en España la adaptación del mismo en su versión normal JS NEO (Ortet et al., 2010) y abreviada JS NEO-S (Ortet et al., 2010).

En lo que respecta al género, son múltiples los estudios que encuentran diferencias significativas entre hombres y mujeres, apreciándose puntuaciones superiores en las mujeres en los factores de Neuroticismo, Apertura a la experiencia y Amabilidad (Balluerka, Gorostiaga, Alonso-Arbiol y Haranburu, 2007; Costa, Terracciano y 
McCrae, 2001; Jonassaint, Siegler, Barefoot, Edwards y Williams, 2011 ), mientras que en el género masculino donde se observaban las puntuaciones más altas, es en el factor Extraversión (Costa, Terracciano y McCrae, 2001). Estas diferencias entre hombres y mujeres se toman como elemento a considerar teniendo en cuenta el grupo de edad. Así, se observan altas puntuaciones en el género femenino en Neuroticismo y Amabilidad a lo largo del ciclo vital, entre hombres y mujeres jóvenes, adultos y mayores (Chapman, Duberstein, Sörensen y Lyness, 2007). Por lo que, a partir de diferentes estudios, se intenta conocer si se producen cambios en los niveles promedio de estas características a lo largo del desarrollo, sin encontrar evidencias al respecto (Roberts, Walton y Viechtbauer, 2008; Terracciano, McCrae, Brant y Costa, 2005; Mossi y Salvatore, 2011), mostrando así, evidencia de la consistencia de la personalidad a lo largo del ciclo vital, como ya exponían McCrae et al. (2000), quienes indicaron que entre los 17 y los 30 años es donde concurre la mayor tasa de cambio en la personalidad, mostrándose estable a partir de los 30 años.

Otro de los aspectos sociodemográficos que son estudiados en relación con las características de personalidad, es el estado civil, observándose que quienes están casados presentan puntuaciones más elevadas en el factor Apertura a la experiencia (Caprara y Steca, 2004). En otros casos, también se analiza la relación del nivel socioeconómico, medido por ejemplo, por el nivel educativo de los padres, con los factores de la personalidad, encontrándose relaciones entre el alto nivel educativo de las madres y altas puntuaciones Extraversión y Apertura, y un bajo nivel educativo de los padres se relaciona con altos niveles de Neuroticismo (Jonassaint et al., 2011).

En España, en los últimos años, son múltiples las investigaciones sobre la personalidad en la última etapa del ciclo vital (Bernarás, Garaigordobil y de las Cuevas, 2011; Gázquez et al., 2009; Hernández, Ehrenzweig y Navarro, 2009; Urquijo, Monchietti, Krzemien, 2009), así como el estudio de los mayores que participan en programas de envejecimiento activo (Gázquez, Pérez-Fuentes y Carrión, 2010). En este contexto, resulta de especial interés, siendo el objetivo de este estudio, analizar las características de la personalidad de un grupo específico de la población de mayores, participantes de un programa de envejecimiento activo como es el Programa Universitario para Personas Mayores de la Universidad de Almería, así como, describir las características de personalidad y analizar ésta, en función de variables como la edad, el género y el estado civil.

\section{METODO}

\section{Participantes}

La muestra está compuesta por 263 personas mayores de 50 años, todos ellos, alumnos de la Universidad de Mayores de la provincia de Almería. En la tabla 1, vemos 
como el $42,6 \%$ son hombres y el $57,4 \%$ son mujeres. En cuanto al estado civil, el 61,2\% de la muestra están casados, más del $30 \%$ son viudos o solteros, y sólo un $16 \%$ están divorciados o separados.

Tabla 1. Distribución de la muestra por género, estado civil y edad

\begin{tabular}{|c|c|c|c|c|c|c|c|c|c|c|c|}
\hline & \multicolumn{2}{|c|}{ Género } & \multicolumn{4}{|c|}{ Estado Civil } & \multicolumn{5}{|c|}{ Edad } \\
\hline & Hombre & Mujer & Casado & Viudo & Soltero & Div/Sep & $<59$ & $60-64$ & $65-69$ & $70-74$ & $>75$ \\
\hline $\mathrm{N}$ & 112 & 151 & 161 & 40 & 42 & 16 & 28 & 36 & 99 & 66 & 34 \\
\hline$\%$ & 42,6 & 57,4 & 61,2 & 15,2 & 16 & 6,1 & 10,6 & 13,7 & 37,6 & 25,1 & 12,9 \\
\hline
\end{tabular}

Por último, quedando reflejado en la misma tabla, la muestra presenta una media de edad de 67 años (DT=6,412); siendo la edad mínima de los participantes de 52 años, y con una máxima de 85 años. En cuanto a la distribución por grupos de edad, observamos unos porcentajes mayoritarios en los grupos de sujetos de 65-69 y 70-74 años, con un $37,6 \%$ y un $25,1 \%$ respectivamente.

\section{Instrumentos}

Cuestionario NEO-FFI (Costa y McCrae, 1999). Una versión reducida del conocido NEO-PI-R, que nos permite la evaluación de la personalidad según el modelo de los "cinco grandes" (Neuroticismo, Extraversión, Apertura, Amabilidad y Responsabilidad) a partir de 60 ítems. Cada pregunta tiene cinco alternativas de respuesta que se puntúan de 0 a 4 ; donde 0 significa "en total desacuerdo", 1 "en desacuerdo", 2 "neutral", 3 "de acuerdo", y 4 "totalmente de acuerdo". De las 60 frases de las que consta la prueba, se utilizan 12 para evaluar cada factor de la personalidad. De este modo, la puntuación máxima que se puede alcanzar en cada factor es de 48, y la mínima de 0.

\section{Procedimiento}

Antes de proceder a la implementación del cuestionario, se solicitaron los permisos necesarios a los responsables del Programa Universitario para Personas Mayores "Ciencia y Experiencia" de la Universidad de Almería, indicando el anonimato de los datos personales y la finalidad de la investigación. Para la aplicación del cuestionario fueron instruidas dos personas que se desplazaron a la sede de Universidad de Mayores de la provincia de Almería, donde se informó al alumnado sobre la finalidad de la investigación, sobre la voluntariedad de la participación, el anonimato de los cambios y posibilidad de asistir a una conferencia donde se iban a dar a conocer los resultados encontrados. La selección de la muestra estuvo compuesta por todos/as los/as alumnos/as que de forma voluntaria quisieron participar en la investigación y que ese día se encontraban en clase. Por lo que del total del alumnado 315 personas, finalmente estaban presentes ese día en clase y aceptaron participar 263 personas. Finalmente, una 
vez fueron implementados los cuestionarios, se procedió a la elaboración de la base de datos, para concluir con el análisis de los mismos.

\section{Análisis de datos}

Análisis Para el análisis de los datos se ha utilizado el programa estadístico SPSS 20. Se trata de un estudio descriptivo, de carácter transversal. Los parámetros descriptivos fueron realizados mediante el análisis de frecuencias y las tablas de contingencia teniendo en cuenta el género. Para la realización de los análisis de puntuaciones medias se aplicó la Prueba $t$ para muestras independientes, con el objetivo de observar si hay diferencias significativas en función del género, y la prueba ANOVA de un factor y las comparaciones múltiples o contrastes post hoc (Scheffé), para analizar las diferencias entre las medias en los distintos estados civiles y grupos de edad. Para conocer el tamaño del efecto aplicamos la $d$ de Cohen para el caso de diferencias entre dos medias, y eta cuadrado, $\mathrm{k}$ medias.

\section{RESULTADOS}

En la tabla 2, observamos como en cuatro de los cinco factores de la personalidad los valores de la media no muestran diferencias significativas entre ambos sexos. Así, sólo en lo que respecta al factor N (neuroticismo), se puede observar cómo las mujeres obtienen puntuaciones significativamente superiores a las de los hombres en este factor $\left(t_{(2,263)}=-4,857 ; p=0,000 ; d=0,61\right)$ siendo considerable el tamaño del efecto de la variable género. Por otro lado, ya sin presentar nivel de significación, los valores promedios entre ambos grupos nos muestran mayores puntuaciones para los hombres en el factor E (extraversión), mientras que las mujeres superan en el factor C (responsabilidad).

Tabla 2. Factores de personalidad y género. Descriptivos y prueba $t$ para muestras independientes

\begin{tabular}{|c|c|c|c|c|c|c|c|c|}
\hline \multirow{3}{*}{ Factor } & \multicolumn{6}{|c|}{ Género } & \multirow{3}{*}{$t$} & \multirow{3}{*}{ Sig. } \\
\hline & \multicolumn{3}{|c|}{ Hombres } & \multicolumn{3}{|c|}{ Mujeres } & & \\
\hline & $N$ & $M$ & $D T$ & $N$ & $M$ & $D T$ & & \\
\hline (N) Neuroticismo & 112 & 15,63 & 7,649 & 151 & 19,93 & 6,670 & $-4,857$ &, $000 * *$ \\
\hline (E) Extraversión & 112 & 29,14 & 6,416 & 151 & 28,69 & 6,063 &, 586 &, 558 \\
\hline (A) Amabilidad & 112 & 27,84 & 6,203 & 151 & 27,74 & 6,889 &, 118 & ,906 \\
\hline (O) Apertura a la experiencia & 112 & 31,68 & 6,644 & 151 & 32,83 & 6,265 & $-1,433$ &, 153 \\
\hline (C) Responsabilidad & 112 & 30,80 & 8,271 & 151 & 31,79 & 5,885 & $-1,074$ & ,284 \\
\hline
\end{tabular}

** La diferencia es significativa al nivel 0.01 
En la tabla 3, se presentan los datos obtenidos según el estado civil de los participantes, tras la aplicación del ANOVA de un factor sólo se dan diferencias significativas en el factor Amabilidad $\left(F_{(3,263)}=6,141, p<0,01\right)$, así, observamos que existen diferencias significativas entre los distintos grupos, aunque siendo el tamaño del efecto pequeño $\left(\eta^{2}=0,067\right)$. Con el fin de conocer entre qué grupos tienen lugar dichas diferencias, se procede a la aplicación de las pruebas post hoc (Scheffé). En el factor A (Amabilidad), el grupo de viudos, son los que obtienen las puntuaciones más bajas, con diferencias significativas con respecto al grupo de los casados y los divorciados/separados.

Tabla 3. Factores de personalidad y estado civil. Descriptivos, ANOVA y post hoc (Scheffé)

\begin{tabular}{|c|c|c|c|c|c|c|c|c|}
\hline \multirow{2}{*}{ Factor } & \multirow{2}{*}{ Estado civil } & \multirow{2}{*}{$N$} & \multirow{2}{*}{$M$} & \multirow{2}{*}{$D T$} & \multicolumn{2}{|c|}{ ANOVA } & \multirow{2}{*}{\multicolumn{2}{|c|}{$\begin{array}{c}\text { Diferencia de medias } \\
\text { (Scheffé) }\end{array}$}} \\
\hline & & & & & $F$ & Sig. & & \\
\hline \multirow{4}{*}{ (N) Neuroticismo } & Soltero (g1) & 40 & 17,30 & 7,401 & \multirow{4}{*}{1,802} & \multirow{4}{*}{, 147 } & \multirow{4}{*}{$\begin{array}{l}\text { g1-g2 } \\
\text { g1-g3 } \\
\text { g1-g4 }\end{array}$} & \multirow{4}{*}{$\mid \begin{array}{l}g 2-g 3 \\
\text { g2-g4 } \\
\text { g3-g4 }\end{array}$} \\
\hline & Casado (g2) & 161 & 18,08 & 7,874 & & & & \\
\hline & Viudo (g3) & 42 & 20,19 & 4,915 & & & & \\
\hline & Div/Sep(g4) & 16 & 15,75 & 7,946 & & & & \\
\hline \multirow{4}{*}{ (E) Extraversión } & Soltero (g1) & 40 & 27,80 & 6,157 & \multirow{4}{*}{1,102} & \multirow{4}{*}{,349 } & \multirow{4}{*}{$\left|\begin{array}{l}g 1-g 2 \\
g 1-g 3 \\
g 1-g 4\end{array}\right|$} & \multirow{4}{*}{$\mid \begin{array}{l}\text { g2-g3 } \\
\text { g2-g4 } \\
\text { g3-g4 }\end{array}$} \\
\hline & Casado (g2) & 161 & 29,48 & 6,684 & & & & \\
\hline & Viudo (g3) & 42 & 28,14 & 4,135 & & & & \\
\hline & Div/Sep(g4) & 16 & 28,50 & 6,218 & & & & \\
\hline \multirow{4}{*}{ (A) Amabilidad } & Soltero (g1) & 40 & 28,00 & 8,302 & \multirow{4}{*}{6,141} & \multirow{4}{*}{, 000} & \multirow{4}{*}{\multicolumn{2}{|c|}{$\begin{array}{l}|\mathrm{g} 1-\mathrm{g} 2||\mathrm{g} 2-\mathrm{g} 3|^{*} \\
|\mathrm{~g} 1-\mathrm{g} 3||\mathrm{g} 2-\mathrm{g} 4| \\
|\mathrm{g} 1-\mathrm{g} 4|\end{array}$}} \\
\hline & Casado (g2) & 161 & 28,04 & 6,350 & & & & \\
\hline & Viudo (g3) & 42 & 24,57 & 3,486 & & & & \\
\hline & Div/Sep(g4) & 16 & 32,25 & 7,946 & & & & \\
\hline \multirow{4}{*}{$\begin{array}{l}\text { (O) Apertura } \\
\text { experiencia }\end{array}$} & Soltero (g1) & 40 & 32,50 & 7,568 & \multirow{4}{*}{1,065} & \multirow{4}{*}{,365 } & \multirow{4}{*}{$\left|\begin{array}{l}g 1-g 2 \\
g 1-g 3 \\
g 1-g 4\end{array}\right|$} & \multirow{4}{*}{$\left|\begin{array}{l}g 2-g 3 \\
g 2-g 4 \\
g 3-g 4\end{array}\right|$} \\
\hline & Casado (g2) & 161 & 32,01 & 6,156 & & & & \\
\hline & Viudo (g3) & 42 & 33,33 & 6,924 & & & & \\
\hline & Div/Sep(g4) & 16 & 34,50 & 4,163 & & & & \\
\hline \multirow{4}{*}{ (C) Responsabilidad } & Soltero (g1) & 40 & 31,80 & 4,592 & & & \multirow{4}{*}{$\mid \begin{array}{l}g 1-g 2 \\
\text { g1-g3 } \\
\text { g1-g4 }\end{array}$} & \multirow{4}{*}{$\left|\begin{array}{l}g 2-g 3 \\
g 2-g 4 \\
g 3-g 4\end{array}\right|$} \\
\hline & Casado (g2) & 161 & 32,02 & 7,895 & \multirow{3}{*}{2,157} & \multirow{3}{*}{,094 } & & \\
\hline & Viudo (g3) & 42 & 29,19 & 6,244 & & & & \\
\hline & Div/Sep(g4) & 16 & 29,75 & 2,955 & & & & \\
\hline
\end{tabular}

En la tabla 4, se analizan las puntuaciones en los cinco factores de la personalidad, con respecto a cinco grupos de edad. Para ello se aplica la ANOVA de un factor que nos muestra diferencias significativas en los factores Extraversión $\left(F_{(4,263)}=11,696, p<0,01\right)$, siendo bajo el tamaño del efecto $\left(\eta^{2}=0,153\right)$; Responsabilidad $\left(F_{(4,263)}=11,105, p<0,01\right)$, siendo bajo el tamaño del efecto $\left(\eta^{2}=0,147\right)$; y disminuyendo un poco más el tamaño del efecto $\left(\eta^{2}=0,115\right)$, también encontramos diferencias significativas respecto al Neuroticismo $\left(F_{(4,263)}=8,400\right.$, $p<0,01)$. Finalmente, siendo el efecto aún menor $\left(\eta^{2}=0,045\right)$, los resultados también muestran diferencias en el factor Apertura $\left(F_{(4,263)}=3,072, p<0,05\right)$.

Nuevamente, con el objetivo de conocer entre qué grupos tienen lugar dichas diferencias, se procede a la aplicación de las pruebas post hoc (Scheffé). Así, 
observamos, respecto al Neuroticismo, que el grupo de 65-69 y el de mayores de 75 años, presenta puntuaciones significativamente mayores respecto a los grupos más jóvenes (< 59 años y 60-64 años) y el grupo de 70-74 años. En el factor Extraversión, es el grupo de 60 a 64 años quién obtiene puntuaciones significativamente mayores respecto a los menores de 59 años, mientras que junto con el grupo de 65 a 69 años, puntuarán significativamente por encima respecto a los dos grupos que engloban a los mayores de 70 años.

En relación al factor Apertura, sólo existen diferencias significativas entre el grupo de 60 a 64 años y los mayores de 75 años, obteniendo, estos últimos puntuaciones estadísticamente inferiores. $\mathrm{Y}$ finalmente, el último de los factores que muestra diferencias significativas es el factor Responsabilidad, observándose cómo en todos los casos es el grupo de mayores de 75 años quienes puntúan significativamente por debajo al resto.

Tabla 4. Factores de personalidad y edad. Descriptivos, ANOVA y post hoc (Scheffé)

\begin{tabular}{|c|c|c|c|c|c|c|c|}
\hline \multirow{2}{*}{ Factor } & \multirow{2}{*}{ Edad } & \multirow{2}{*}{$N$} & \multirow{2}{*}{$M$} & \multirow{2}{*}{$D T$} & \multicolumn{2}{|c|}{ ANOVA } & \multirow{2}{*}{ Diferencia de medias (Scheffé) } \\
\hline & & & & & $F$ & Sig. & \\
\hline \multirow{5}{*}{$(\mathrm{N})$} & $<59(\mathrm{~g} 1)$ & 28 & 15,00 & 7,463 & \multirow{5}{*}{8,400} & \multirow{5}{*}{, 000 } & \multirow{5}{*}{ 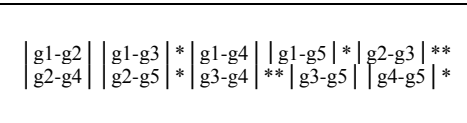 } \\
\hline & 60-64 (g2) & 36 & 15,22 & 6,672 & & & \\
\hline & $65-69$ (g3) & 99 & 20,41 & 6,775 & & & \\
\hline & 70-74 (g4) & 66 & 16,03 & 7,567 & & & \\
\hline & $>75(\mathrm{~g} 5)$ & 34 & 20,94 & 6,564 & & & \\
\hline \multirow{5}{*}{ (E) } & $<59(\mathrm{~g} 1)$ & 28 & 28,00 & 6,418 & \multirow{5}{*}{11,696} & \multirow{5}{*}{,000 } & \multirow{5}{*}{ 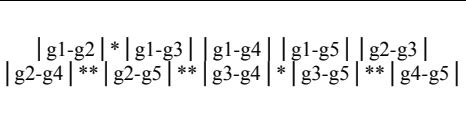 } \\
\hline & 60-64 (g2) & 36 & 33,11 & 3,429 & & & \\
\hline & $65-69$ (g3) & 99 & 30,18 & 6,577 & & & \\
\hline & $70-74$ (g4) & 66 & 26,88 & 5,302 & & & \\
\hline & $>75(\mathrm{~g} 5)$ & 34 & 25,24 & 5,388 & & & \\
\hline \multirow{5}{*}{ (A) } & $<59(\mathrm{~g} 1)$ & 28 & 28,00 & 3,611 & \multirow{5}{*}{1,711} & \multirow{5}{*}{,148 } & \multirow{5}{*}{$\left|\begin{array}{l}\text { g1-g2 } \\
\text { g2-g4 }\end{array}\right|\left|\begin{array}{l}\text { g1-g3 } \\
\text { g2-g5 }\end{array}\right|\left|\begin{array}{l}\text { g1-g4 } \\
\text { g3-g4 }\end{array}\right|\left|\begin{array}{l}\text { g1-g5 } \\
\text { g3-g5 }\end{array}\right|$\begin{tabular}{|l} 
g2-g3 \\
g4-g5
\end{tabular} \mid} \\
\hline & 60-64 (g2) & 36 & 29,11 & 7,034 & & & \\
\hline & $65-69$ (g3) & 99 & 28,41 & 6,289 & & & \\
\hline & $70-74$ (g4) & 66 & 27,15 & 7,958 & & & \\
\hline & $>75(\mathrm{~g} 5)$ & 34 & 25,59 & 5,560 & & & \\
\hline \multirow{5}{*}{ (O) } & $<59(\mathrm{~g} 1)$ & 28 & 33,29 & 5,408 & \multirow{5}{*}{3,072} & \multirow{5}{*}{,017 } & \multirow{5}{*}{ 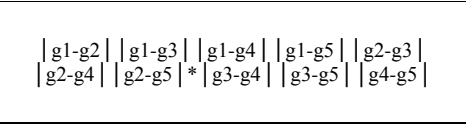 } \\
\hline & $60-64(\mathrm{~g} 2)$ & 36 & 33,67 & 4,085 & & & \\
\hline & $65-69$ (g3) & 99 & 32,70 & 7,306 & & & \\
\hline & $70-74$ (g4) & 66 & 32,42 & 5,881 & & & \\
\hline & $>75(\mathrm{~g} 5)$ & 34 & 28,94 & 6,800 & & & \\
\hline \multirow{5}{*}{ (C) } & $<<59(\mathrm{~g} 1)$ & 28 & 30,71 & 5,381 & \multirow{5}{*}{11,105} & \multirow{5}{*}{,000 } & \multirow{5}{*}{ 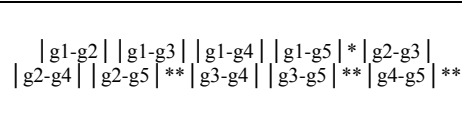 } \\
\hline & 60-64 (g2) & 36 & 32,56 & 7,315 & & & \\
\hline & $65-69$ (g3) & 99 & 32,75 & 5,932 & & & \\
\hline & 70-74 (g4) & 66 & 32,42 & 7,091 & & & \\
\hline & $>75(\mathrm{~g} 5)$ & 34 & 24,59 & 6,946 & & & \\
\hline
\end{tabular}

\section{DISCUSION}

A través de la aplicación del NEO-FFI, hemos podido conocer las características de las personalidad de un grupo específico de mayores de 50 años, que forman parte de un programa de envejecimiento activo, como es, el Programa Universitario para Personas Mayores "Ciencia y Experiencia" de la Universidad de Almería. 
Así, respecto al género, los resultados nos muestran que las mujeres obtienen puntuaciones significativamente superiores a las de los hombres en el factor Neuroticismo, siendo considerable el tamaño del efecto del género en dicha diferencia $(d=0,61)$ y escasamente superior al encontrado por Balluerka, Gorostiaga, AlonsoArbiol y Haranburu (2007), y coincidiendo con los resultados obtenidos en el estudio transcultural del género realizado por Costa, Terracciano y McCrae (2001); Chapman et al. (2007), y otros estudios como el de Jonassaint et al. (2011), aunque en nuestro caso no se aprecian diferencias significativas en otros factores, tales como, Amabilidad y Apertura a la experiencia. Así, en este último factor, es donde encontramos la única diferencia significativa entre hombres y mujeres, estando estas últimas por encima en estudios que analizan específicamente las características de personalidad de este grupo específico de personas, que forman parte de los programas de envejecimiento activo de los Programas para mayores (Bernarás, Garaigordobil y de las Cuevas, 2011).

Por otro lado, en el análisis de las puntuaciones de los hombres, no observamos ninguna diferencia significativa, mientras que otros estudios indican, que es el factor Extraversión donde se observaban puntuaciones más altas para los hombres, (Costa, Terracciano y McCrae, 2001). Así, en nuestro caso, aunque las diferencias no son significativas, este es uno de los factores, junto con el factor Amabilidad, donde los hombres obtienen medias superiores a las mujeres.

Estas discrepancias respecto al género, en los diferentes estudios, confirman la hipótesis propuesta por Costa y McCrae $(1985,1992)$ sobre que éste, sería una variable de influencia social y/o cultural, en lo que a la presencia de factores de la personalidad se refiere.

Respecto al estado civil, no se advierten diferencias entre los grupos, salvo en el factor Amabilidad, que los viudos presentan puntuaciones más bajas, con diferencias significativas con respecto al grupo de los casados y los divorciados/separados. Algunos estudios apuntan a que la influencia del estado civil se puede ver influenciada por aspectos más relacionados con el apoyo social (Caprara y Steca, 2004), y debemos tener en cuenta, que se trata de un grupo especial dentro de la población general de mayores, ya que uno de los objetivos que persiguen estos programas, es favorecer la interacción social de los mayores, así, esta limitación del estudio, se eliminaría contrastando los resultados de este grupo, con otro de mayores que no formaran parte de este programa, para conocer si estas diferencias en función del estado civil se mantienen en ambos grupos.

Por último, respecto a los grupos de edad, observamos que existen diferencias significativas respecto al factor Extraversión, Responsabilidad, Neuroticismo y Apertura, aunque la proporción de variabilidad total de la puntuación obtenida en cada uno de estos factores atribuible a la variable edad es baja. Por ello, a pesar de que existan diferencias entre los grupos, en cierto modo, esto viene a corroborar la hipótesis de la 
estabilidad de la personalidad en los adultos y mayores (McCrae et al., 2000; Roberts, Walton y Viechtbauer, 2006; Terracciano, McCrae, Brant y Costa, 2005), aunque concretamente, en nuestro estudio, esto puede afirmarse sobre todo para el factor Amabilidad, donde los diferentes grupos etarios no presentan diferencias significativas en su puntuación media.

\section{REFERENCIAS}

Balluerka, N., Gorostiaga, A., Alonso-Arbiol, I. y Haranburu, M. (2007). La adaptación de instrumentos de medida de unas culturas a otras: una perspectiva práctica. Psicothema, 19(1), 124-133.

Bernarás, E., Garaigordobil, M. y de las Cuevas, C. (2011). Inteligencia emocional y rasgos de personalidad. Influencia de la edad y el género durante la edad adulta y la vejez. Boletín de Psicología, 103, 75-88.

Caprara, M. y Steca, P. (2004). Personalidad y envejecimiento. Intervención Psicosocial, 13(1), 85-98.

Cattell, R.B. (1949). An introduction to personality study. London: Hutchinson.

Cattell, R.B. (1993). Sixteen Personality Factor Questionaire, Fifth Edition. Champaign, IL: Institute for Personality and Ability Testing, Inc.

Chapman, B.P., Duberstein, P.R., Sörensen, S. y Lyness, J.M. (2007). Gender differences in Five Factor Model personality traits in an elderly cohort. Personality and Individual Differences, 43, 1594-1603.

Costa, P.T. y McCrae, R.R. (1985). The NEO Personality Inventory Manual. Odessa, FL: Psychological Assessment Resources.

Costa, P.T. y McCrae, R.R. (1989). The NEO-PI/NEO-FFI manual supplement. Odessa, FL: Psychological Assessment Resources.

Costa, P.T. y McCrae, R.R. (1992). NEO-PI-R. Professional Manual. Odessa, FL: Psychological Assessment Resources.

Costa, P.T., McCrae, R.R. y Martin, T.A. (2008). Incipient adult personality: The NEO-PI-3 in middle-school-aged children. British Journal of Developmental Psychology, 26, 71-89.

Costa, P.T., Terracciano, A. y McCrae, R.R. (2001). Gender differences in personality traits across cultures. Robust and surprising findings. Journal of Personality and Social Psychology, 81(2), 322-331.

De Miguel, A. (2005). Diferencias de edad y género en el NEO-PI-R en dos muestras con distinto nivel académico. International Journal of Psychology and Psychological Therapy, 1(1), 13-31.

Gázquez, J.J., Pérez-Fuentes, M.C. y Carrión, J.J. (2010). Análisis de la Memoria Cotidiana en alumnos del Programa Universitario para Mayores en Almería. European Journal of Education and Psychology, 3(1), 155-165.

Gázquez, J.J., Pérez-Fuentes, M.C., Fernández, M., González, L., Ruiz, I. y Díaz, A. (2009). Estereotipos sobre la vejez y su relación con la formación en gerontología: un estudio integernacional. European Journal of Education and Psychology, 2(3), 263-273.

Hernández, Z.E., Ehrenzweig, Y. y Navarro, A.M. (2009). Factores psicológicos, demográficos y sociales asociados al estrés y a la Personalidad Resistente en adultos mayores. Pensamiento Psicológico, 5(12), 13-28. 
Jonassaint, C.R., Siegler, I.C., Barefoot, J.C., Edwards, C.L. y Williams, R.B. (2011). Low Life Course Socioeconomic Status (SES) is Associated with Negative NEO PI-R Personality Patterns. International Society of Behavioral Medicine, 18, 13-21.

McCrae, R.R. y Costa, P.T. (2007). Brief versions of the NEO-PI-3. Journal of Individual Differences, 28, 116-128.

McCrae, R.R. y Costa, P.T. (2008). Empirical and theoretical status of the Five-Factor Model of personality traits. En G.J. Boyle, G. Matthews y D.H. Sakloske (Eds.), Personality theory and assessment. Vol. 1. Personality theories and models (pp. 273-294). Londres: SAGE.

McCrae, R.R., Costa, P.T. y Martin, T.A. (2005). The NEO-PI-3: a more readable revised NEO Personality Inventory. Journal of Personality Assessment, 84, 261-270.

McCrae, R.R., Costa, P.T., Ostendorf, F., Angleitner, A., Avia, M.D., Sanz, J., SánchezBernardos, M.L., Kusdil, M.E., Woodfield, R., Saunders, P.R. y Smith, P.B. (2000). Nature over nurture: temperament, personality and life span development. Journal of Personality and Social Psychology, 78, 173-186.

McCrae, R.R., Martin, T.A. y Costa, P.T. (2005). Age Trends and Age Norms for the NEO Personality Inventory-3 in Adolescents and Adults. Assessment, 12(4), 363-373.

McCrar, R.R. y Terracciano, A. (2005). Universal Features of Personality Traits From the Observer's Perspective: Data From 50 Cultures. Journal of Personality and Social Psychology, 88(3), 547-561.

McDougall, W. (1932). Of the words character and personality. Journal of Personality, 1(1), 3-16.

Mossi, P. y Salvatore, S. (2011). Psychological transition from meaning to sense. European Journal of Education and Psychology, 4(2), 153-169.

Norman, W.T. (1963). Toward an adequate taxonomy of personality attributes: replicated factor structure in peer nomination personality ratings. The Journal of Abnormal and Social Psychology, 66(6), 574-583.

Ortet, G., Escrivá, P., Ibáñez, M.I., Moya, J., Villa, H., Mezquita, L. y Ruipérez, M.A. (2010). Versión corta de la adaptación española para adolescentes del NEO-PI-R (JS NEO-S). International Journal of Clinical and Health Psychology, 10(2), 327-344.

Ortet, G., Ibáñez, M.I., Ruipérez, M.A., Villa, H., Moya, J. y Escrivá, P. (2010). Adaptación para adolescentes de la versión española del NEO PI-R (JS NEO). Psicothema, 19(2), 263268.

Roberts, B.W., Walton, K.E. y Viechtbauer, W. (2008). Patterns of mean-level change in personality traits across the life course: A meta-analysis of longitudinal studies. Psychological Bulletin, 132, 1-25.

Salguero, J.M., Fernández-Berrocal, P., Ruiz-Aranda, D., Castillo, R. y Palomera, R. (2011). Inteligencia emocional y ajuste psicosocial en la adolescencia: El papel de la percepción emocional. European Journal of Education and Psychology, 4(2), 143-152.

Terracciano, A., McCrae, R.R., Brant, L.J. y Costa, P.T. (2005). Hierarchical linear modeling analyses of the NEO-PI-R scales in the Baltimore Longitudinal Study of Aging. Psychology and Aging, 20, 493-506.

Urquijo, S., Monchietti, A. y Krzemien, D. (2009). Adaptación a la crisis vital del envejecimiento: rol de los estilos de personalidad y de la apreciación cognitiva en adultas mayores. Anales de Psicología, 24(2), 299-311. 